\title{
Why should breast surgeons use ultrasound?
}

\author{
M. Ahmed $\cdot$ N. Abdullah $\cdot$ S. Cawthorn • \\ S. I. Usiskin • M. Douek
}

Received: 27 February 2014/ Accepted: 17 March 2014/Published online: 5 April 2014

(C) Springer Science+Business Media New York 2014

\begin{abstract}
Portable ultrasound is now used in a variety of clinical settings by specialties outside of radiology. Despite increased accessibility to ultrasound, the overall performance of ultrasound by breast surgeons is consistently low. We discuss the reasons why this is unacceptable for future patient care and answer the question, 'Why should breast surgeons use ultrasound?' We reviewed the literature for evidence assessing the outcomes of breast surgeon-performed ultrasound both intra-operatively and in the outpatient department. Intra-operative ultrasound performed by surgeons reduces re-excision rates in breast-conserving surgery. Outpatient-based ultrasound performed by surgeons frees up the resources of radiology departments, allowing them to focus upon patients requiring more complex diagnostic and interventional procedures. For surgeons to competently perform intra-operative and outpatient-based ultrasound, a period of formal ultrasound
\end{abstract}

M. Ahmed · M. Douek ( $\square)$

Department of Research Oncology, King's College London,

Guy's Hospital Campus, 3rd Floor Bermondsey Wing, Great

Maze Pond, London SE1 9RT, UK

e-mail:michael.douek@kcl.ac.uk

M. Ahmed

e-mail: muneer.ahmed@kcl.ac.uk

N. Abdullah

Division of Breast Reconstructive Surgery, Department of Surgery, Universiti Kebangsaan Malaysia Medical Centre, 56000 Kuala Lumpur, Malaysia

S. Cawthorn

Department of Breast Surgery, Frenchay Hospital, North Bristol NHS Trust, Bristol BS16 1LE, UK

\section{S. I. Usiskin}

Department of Breast Radiology, St Bartholomew's Hospital, London, UK training is necessary to acquire knowledge of ultrasound skills and techniques. This should be followed by a period of mentorship and supervised training with an experienced breast radiologist. Breast surgeon-performed ultrasound is beneficial to the multi-disciplinary care of breast cancer patients. To further improve multidisciplinary care, breast surgeons and radiologists should work more collaboratively to optimise imaging applications both in the operating theatre and outpatient department. Current advances in therapeutic percutaneous techniques are of interest to both surgeons and radiologists. In future, a hybrid specialisation should be considered to incorporate accreditation in both specialties for breast interventional procedures.

Keywords Intra-operative ultrasound - Surgeonperformed ultrasound $\cdot$ Breast-conserving surgery

\section{Ultrasound performed by breast surgeons}

Technological advances have reduced the size of ultrasound scanners, making them portable, practical and able to be used close to the bed-side or in the outpatient department. Portable ultrasound examination can be considered as simply an extension of clinical examination. Ultrasound is essential in the performance of diagnostic and interventional procedures in breast disease. Despite, the benefits of ultrasound in the management of breast disease, its utilisation by breast surgeons is very variable. A prospective cohort analysis of the use of breast ultrasound amongst 126 Australian and New Zealand breast surgeons [1] demonstrated that of the 59 responding surgeons, only $47 \%$ had full-time access to an ultrasound scanner, with a further $30 \%$ being reliant upon opportunistic shared availability. A total of $63 \%$ performed at least one 
ultrasound scan per month but only $39 \%$ performed more than 10 , and $50 \%$ of all responders stated that they did not perform ultrasound-guided biopsies. The prospective cohort survey of the American College of Surgeons ultrasound education programme [2] analysing 873 responders (49\% response rate) identified that over one-third of surgeons who attended the programme were unable to perform ultrasound examinations. The main reasons for this were related to their radiology department with almost half stating that radiologists had prohibited them from scanning, the remainder being due to a combination of a lack of time, hospital restrictions, lack of confidence and reimbursement as well as medico-legal liability [2].

Departmental ultrasound has also improved with the introduction of functions such as doppler and harmonic imaging. There is a clinical need for high resolution imaging in the context of triple assessment of patients with suspected breast cancer and for targeted imaging after positive findings on digital mammography and increasingly, breast magnetic resonance imaging (MRI). Characterization of breast lesions is best undertaken by radiologists, using a departmental ultrasound. This is quite distinct from the clinical application of portable ultrasound at the bed-side as an extension of clinical examination and in order to localise in situ, lesions that have already been characterised by breast radiologists.

\section{Role of intra-operative ultrasound}

This lack of comprehensive use of ultrasound by breast surgeons gains clinical significance in view of the increasing evidence of the role of intra-operative ultrasound (IOUS) in improving the outcomes of breast-conserving surgery [3, 4]. Krekel et al. [4] in their randomised controlled trial (RCT) of ultrasound-guided versus palpation-only surgery during breast-conserving surgery for palpable breast cancer, demonstrated that the use of IOUS was able to significantly reduce the tumour involved margin rates, re-excision rates and the volume of excised specimens compared to surgery by palpation alone. The successful application of IOUS is dependent upon a combination of technique and experience to be able to utilise ultrasound during tumour excision. Such skills can only be acquired by supervised training with a breast radiologist, and all surgeons involved in the study by Krekel et al. [4] underwent an ultrasound-training programme performing ten cases, under strict supervision of a breast radiologist [5]. Within these ten cases the surgeons had easily familiarised themselves with the technique, rapidly mastered basic ultrasound skills and showed proficiency in performing IOUS. This suggests that the learning curve is small, but dependent upon a close working relationship with the radiology department for their initial input within the short learning phase. Krekel et al. [5] also demonstrated that the greater prior experience of surgeons in the use of ultrasound, the faster their ability to become proficient with IOUS during the learning phase, [5] adding weight to surgeons performing outpatient-based ultrasound to enhance IOUS proficiency.

The benefit of IOUS is not confined to palpable breast cancer alone. Ahmed et al. [3] in a meta-analysis of IOUS versus wire-guided localization (WGL) in the treatment of non-palpable breast cancer, found a significant reduction in the involved surgical margin rate within the IOUS group. With only one out of ten included studies being an RCT, no significant difference between the IOUS and WGL groups with respect to in situ disease was identified, suggesting that a bias towards surgeon selection of micro-calcifications to the WGL group was not present. Krekel et al. [6] also performed a three-way prospective cohort study of 201 patients comparing IOUS, WGL and radioguided occult lesion localization in the treatment of non-palpable breast cancers. The authors demonstrated that IOUS significantly reduced the involved surgical margin rate when compared with the other two procedures. With greater than $90 \%$ of WGL being performed under ultrasound guidance, [3] in these cases, it would be possible to replace WGL with IOUS. This would avoid the need for radiology input for the localisation of many non-palpable lesions on the day of surgery once the lesion has been characterised, preventing scheduling conflicts and allowing radiologists to focus on more complex diagnostic and interventional procedures.

IOUS is not only limited to the surgical excision of breast cancer. The role of intra-operative radiotherapy (IORT) for selected patients with breast cancer [7, 8] is emerging, and its successful implementation is also dependent upon the use of ultrasound. The skin dose from IORT is dependent upon the distance between the IORT source and the skin and therefore great care must be taken in order to ensure that a safe distance is maintained to reduce IORT-related skin complications. [9] The only intra-operative imaging modality to measure skin flap thickness is ultrasound, and this may prove to be useful in other areas of breast surgery.

\section{Outcomes of ultrasound performed by breast surgeons}

Evidence exists to support the role of IOUS in improving patient outcomes in breast-conserving surgery, $[3,4,6]$ but the utilisation of IOUS amongst breast surgeons remains consistently low, with surveys of American and Australasian breast surgeons suggesting figures between 2.8 and $17 \%$, respectively $[1,2]$. This low utilisation of IOUS is likely reflective of surgeons not receiving formal, 
structured training, [1] with figures of between 22 to $55 \%$ of residency programmes providing formal training in ultrasound [10]. This deficiency in ultrasound training is not only confined to residency programmes but also senior breast fellowships where trainees consistently report inadequacies in ultrasound training [11]. It has been shown that even short courses of structured theoretical and practical skills teaching have significantly improved breast ultrasound skills amongst surgeons [12]. Donaldson et al. [13] in their prospective series of 551 surgeon performed ultrasound-guided core biopsies identified a false negative rate of $1.2 \%$. Whitehouse et al. [14] performed a prospective cohort study on 231 patients who underwent ultrasound assessment by both radiologist and surgeon and definitive histopathological confirmation of diagnosis. Whitehouse et al. [14] identified only a $3.9 \%$ complete discordance between radiologists and surgeons. In the discordant scans, the surgeon was correct in seven of eight cases and the radiologist in only one of eight, with the radiologist incorrectly identifying five cases that were malignant on histology as benign, with the surgeon not missing any malignant cases. This strongly supports that basic diagnostic ultrasound skills can be taught to surgeons within a relatively short time frame and resulting in excellent outcomes. These transferable diagnostic skills can subsequently be applied to the performance of IOUS, shortening the learning curve even further [4]. Currently, within the United Kingdom formal ultrasound training is not included within general surgery, or the breast subspecialty (oncoplastic surgery) interest training programme. Breast radiologists invariably utilise departmental ultrasound, and there is limited evidence of the use of portable ultrasound by breast radiologists. The Royal College of Radiologists [15] of the United Kingdom has established competencies in breast ultrasonography and ultrasoundguided core biopsies for non-radiologists, accepting its safe utilisation by other specialties and ensuring that high standards are maintained.

\section{Outpatient-based ultrasound}

The management of breast cancer is increasingly being centralised in tertiary facilities, which have readily available access to breast radiologists for diagnostic ultrasound and performance of ultrasound-guided biopsies. The proposal that breast surgeons develop ultrasound skills does not automatically equate with shifting the balance of the imaging workload in places with adequately resourced radiology departments. However, in conducting outpatientbased ultrasound and interventions, it would allow surgeons to develop and maintain their ultrasound skills, which could then be applied to IOUS. Surgeons would be able to closely monitor the response of patients to primary systemic therapy within the clinic allowing radiologists to focus on interpretation of more complex imagings such as MRI. Surgeons could provide triple assessment of palpable lesions and therapeutic intervention of benign lesions such as aspiration of symptomatic cysts or abscesses, clearly visible on ultrasound in clinic without burdening the radiology department unnecessarily. Sharing the imaging workload would also free up the resources of overstretched radiology departments to focus upon patients requiring more complex diagnostic and interventional procedures [13] and potentially reducing their waiting times. Equally, the continually increasing demand for breast services [16] means hospitals with less resourced radiology departments would benefit from surgeons trained in ultrasound being able to perform routine triple assessment independently when radiologists are not available, to help reduce waiting times for patients. Emerging evidence may prove that outpatient ultrasound, in selected patients, provides definitive staging of the axilla in breast cancer and eradicates the need for the more invasive, resource-demanding sentinel lymph node biopsy, [17] which is radioisotope dependent and consequently inaccessible to $40 \%$ of breast cancer patients in the developed world [18]. The use of ultrasound in the clinic would allow for meticulous pre-operative planning by marking the size and position of lesions accurately, determining the most appropriate incision and the ability to perform skin-sparing surgery depending upon the depth of the lesion from the skin. Such crucial aspects of surgery could be shared with the patient in the clinic, rather than only after surgery. In performing outpatientbased ultrasound, it is essential that surgeons be open to audit of their outcomes as would be expected of any radiology department performed imaging, in order to maintain the highest standards of clinical excellence.

\section{The future}

The best evidence base available demonstrates the benefits of IOUS in breast-conserving surgery, and this has to be recognised. Adequate ultrasound training should be included in the surgical curriculum for breast surgical trainees. Such training should consist of a structured training programme combining formal theoretical and practical skills teaching with a fixed radiology placement, [1, 12, 19] which would allow the trainee the opportunity to consolidate their basic ultrasound skills for lesion identification through adequate caseload exposure under the supervision of an experienced breast radiologist. The roles of the breast surgeon and radiologist will likely change with the increasing application of intra-operative imaging and minimally invasive ablative techniques for breast cancer 
treatment. Whilst previous generations of radiologists often underwent surgical training and read for Membership Examinations of the Royal Colleges of Surgeons in the UK and Ireland before embarking upon their radiology training, the current generation of radiology trainees now enter into seamless 'run-through' training, without such surgical exposure [20]. In future, breast surgery trainees should receive more detailed and practical 'core' knowledge in radiology, and radiology trainees should receive 'core' surgical knowledge. This would allow the development of future breast specialists who have a better understanding of the working environments of their colleagues and who are able to embrace both surgical and radiological techniques and apply the best evidence-based practice to the care of their patients.

Conflict of interest The authors have no disclosures to make concerning financial and personal relationships with other people or organisations that could inappropriately influence their work. No ethical approval was requested for this work.

\section{References}

1. Law MT, Kollias J, Bennett I (2013) Evaluation of office ultrasound usage among Australian and New Zealand breast surgeons. World J Surg 37(9):2148-2154

2. Staren ED, Knudson MM, Rozycki GS, Harness JK, Wherry DC, Shackford SR (2006) An evaluation of the American College of Surgeons' ultrasound education program. Am J Surg 191(4):489-496

3. Ahmed M, Douek M (2013) Intra-operative ultrasound versus wire-guided localization in the surgical management of nonpalpable breast cancers: systematic review and meta-analysis. Breast Cancer Res Treat 140(3):435-446

4. Krekel NM, Haloua MH, Lopes Cardozo AM, de Wit RH, Bosch AM, de Widt-Levert LM, Muller S, van der Veen H, Bergers E, de Lange Klerk ES et al (2013) Intraoperative ultrasound guidance for palpable breast cancer excision (COBALT trial): a multicentre, randomised controlled trial. The lancet oncology 14(1):48-54

5. Krekel NM, Lopes Cardozo AM, Muller S, Bergers E, Meijer S, van den Tol MP (2011) Optimising surgical accuracy in palpable breast cancer with intra-operative breast ultrasound-feasibility and surgeons' learning curve. Eur J Surg Oncol 37(12): $1044-1050$
6. Krekel NM, Zonderhuis BM, Stockmann HB, Schreurs WH, van der Veen H, de Lange Klerk ES, Meijer S, van den Tol MP (2011) A comparison of three methods for nonpalpable breast cancer excision. Eur J Surg Oncol 37(2):109-115

7. Vaidya JS, Wenz F, Bulsara M, Tobias JS, Joseph DJ, Keshtgar M, Flyger HL, Massarut S, Alvarado M, Saunders C et al (2013) Risk-adapted targeted intraoperative radiotherapy versus wholebreast radiotherapy for breast cancer: 5-year results for local control and overall survival from the TARGIT-A randomised trial. Lancet 383(9917):603-613

8. Veronesi U, Orecchia R, Maisonneuve P, Viale G, Rotmensz N, Sangalli C, Luini A, Veronesi P, Galimberti V, Zurrida S et al (2013) Intraoperative radiotherapy versus external radiotherapy for early breast cancer (ELIOT): a randomised controlled equivalence trial. Lancet Oncol 14(13):1269-1277

9. Smith BD, Arthur DW, Buchholz TA, Haffty BG, Hahn CA, Hardenbergh PH, Julian TB, Marks LB, Todor DA, Vicini FA et al (2009) Accelerated partial breast irradiation consensus statement from the American Society for Radiation Oncology (ASTRO). Int J Radiat Oncol Biol Phys 74(4):987-1001

10. Freitas ML, Frangos SG, Frankel HL (2006) The status of ultrasonography training and use in general surgery residency programs. J Am Coll Surg 202(3):453-458

11. Sclafani LM, Bleznak A, Kelly T, El-Tamer MB (2012) Training a new generation of breast surgeons: are we succeeding? Ann Surg Oncol 19(6):1856-1861

12. Law MT, Bennett IC (2010) Structured ultrasonography workshop for breast surgeons: is it an effective training tool? World J Surg 34(3):549-554

13. Donaldson LA, Cliff A, Gardiner L, Hubbard AE, Ashton MA (2003) Surgeon-controlled ultrasound-guided core biopsies in the breast-a prospective study and a new use for surgeons in the clinic. Eur J Surg Oncol 29(2):139-142

14. Whitehouse PA, Baber Y, Brown G, Moskovic E, King DM, Gui GP (2001) The use of ultrasound by breast surgeons in outpatients: an accurate extension of clinical diagnosis. Eur J Surg Oncol 27(7):611-616

15. Royal College of Radiologists. Ultrasound Training for Medical and Surgical Specialties. < http://www.rcr.ac.uk>

16. Dehn T (2008) Who should run breast clinics, surgeons or radiologists? Ann R Coll Surg Engl 90(3):187-192

17. Gentilini O, Veronesi U (2012) Abandoning sentinel lymph node biopsy in early breast cancer? A new trial in progress at the European Institute of Oncology of Milan (SOUND: sentinel node vs Observation after axillary UltraSouND). Breast 21(5):678-681

18. Rescigno J, Zampell JC, Axelrod D (2009) Patterns of axillary surgical care for breast cancer in the era of sentinel lymph node biopsy. Ann Surg Oncol 16(3):687-696

19. Gresens AA, Britt RC, Feliberti EC, Britt LD (2012) Ultrasoundguided breast biopsy for surgical residents: evaluation of a phantom model. J Surg Educ 69(3):411-415

20. http://specialtytraining.hee.nhs.uk. Accessed 1 Feb 2014 